\title{
Detectable dimension-6 proton decay in SUSY SO(10) GUT at Hyper-Kamiokande
}

\author{
Naoyuki Haba, Yukihiro Mimura and Toshifumi Yamada \\ Institute of Science and Engineering, Shimane University, \\ 1060 Nishi-Kawatsu, Matsue City, Shimane, 690-8504, Japan \\ E-mail: haba@riko.shimane-u.ac.jp, mimura@riko.shimane-u.ac.jp, \\ toshifumi@riko.shimane-u.ac.jp
}

ABSTRACT: In the minimal SUSY SU(5) GUT with $O(1)$ TeV SUSY particles and $O(1)$ or below self-coupling for the GUT-breaking Higgs field, the width of the dimension-6 proton decay is suppressed below the reach of Hyper-Kamiokande. In this paper, we point out that a SUSY SO(10) GUT which adopts only $\mathbf{4 5}_{\mathrm{H}}+\mathbf{1 6}_{\mathrm{H}}+\overline{\mathbf{1 6}}_{\mathrm{H}}$ GUT-breaking Higgs fields leads to an enhanced dimension- 6 proton decay width detectable at Hyper-Kamiokande. The enhancement is because the SU(5)-breaking VEV of $\mathbf{4} \mathbf{5}_{\mathrm{H}}$ arises due to Planck-suppressed terms, $W \propto\left(\mathbf{4 5} 5_{\mathrm{H}}^{2}\right)^{2} / M_{*}+\mathbf{4 5} 5_{\mathrm{H}}^{4} / M_{*}$, and is therefore substantially larger than the other VEVs that conserve $\mathrm{SU}(5)$. As a result, the $(\mathbf{3}, \mathbf{2}, 1 / 6)$ GUT gauge boson mass is about $1 / 5$ smaller than the $(\mathbf{3}, \mathbf{2},-5 / 6)$ GUT gauge boson mass and can induce a fast dimension- 6 proton decay. Through a numerical analysis on threshold corrections of the GUT gauge bosons and the physical components of the GUT-breaking Higgs fields, we confirm that the dimension-6 proton decay can be within the reach of Hyper-Kamiokande.

KEYwORDS: GUT, Supersymmetric Standard Model

ArXiv EPRINT: 1904.11697 


\section{Contents}

1 Introduction $\quad 1$

2 Spectrum of the $\mathrm{SO}(10)$ gauge bosons and proton decay 3

$\begin{array}{lll}3 & \mathrm{SO}(10) & \text { breaking vacua in the model }\end{array}$

4 GUT-scale threshold corrections for the gauge coupling unification $\quad 5$

$\begin{array}{lll}5 & \text { Numerical result } & 7\end{array}$

6 Conclusion 10

$\begin{array}{ll}\text { A Mass spectrum } & 10\end{array}$

B Renormalizable model obtained by employing $54_{\mathrm{H}} \quad 11$

\section{Introduction}

The dimension-6 proton decay is an important prediction of the grand unified theory (GUT) [1]. The Super-Kamiokande experiment currently gives the bound of the partial proton lifetime $\tau\left(p \rightarrow \pi^{0} e^{+}\right)>1.6 \times 10^{34}$ years (90\% confidence level) [2], and it will be searched up to $6.3 \times 10^{34}$ years at $3 \sigma$ level by a 10 year exposure of one 187 kton fiducial volume detector at Hyper-Kamiokande (HK) [3]. Now that the HK experiment is scheduled to start in 2026, it is time to survey GUT models which predict the dimension- 6 proton decay within the discovery reach.

In the minimal supersymmetric (SUSY) SU(5) GUT [4-6] with $O(1) \mathrm{TeV}$ SUSY particles, the partial lifetime for the dimension-6 proton decay via GUT gauge boson exchange is predicted to be more than a few times $10^{35}$ years naively. The gauge coupling unification condition does not directly give the mass of the GUT gauge boson, because the mass of the physical components of the SU(5)-breaking Higgs field $\mathbf{2 4}_{\mathrm{H}}$ cannot be determined theoretically. The GUT gauge boson mass becomes heavier (the proton lifetime is longer) if the self-coupling of $\mathbf{2 4}_{\mathrm{H}}$ is smaller. ${ }^{1}$ The SUSY GUTs also predict the dimension-5 proton decay via colored Higgs exchange [7,8], such as $p \rightarrow K^{+} \bar{\nu}$, whose current bound reads $\tau\left(p \rightarrow K^{+} \bar{\nu}\right)>5.9 \times 10^{33}$ years [9] and which often gives a severe constraint on the model construction. There are several ways to suppress the dimension-5 decay to a harmless level, e.g., by enhancing the colored Higgs mass with SUSY particle threshold with large

\footnotetext{
${ }^{1}$ In non-SUSY SU(5) GUTs, some choices of GUT Higgses yield models that survive the SK bound and will be explored at HK [17].
} 
wino/gluino mass ratio [10], with non-renormalizable superpotential of adjoint representations [11], or with GUT particle thresholds in non-minimal models for the gauge coupling evolutions [12]. Other ways include assuming heavy squarks, or utilizing a cancellation among multiple Higgs couplings. Compared to the dimension- 5 decay, the dimension- 6 proton decay involves less parameters and its naive prediction is above the current experimental bound. Therefore, it is worth pursuing the possibility that $p \rightarrow \pi^{0} e^{+}$will be observed at HK. In fact, as the LHC results imply that the SUSY particles have mass above multi-TeV scale, some people revisit the unification conditions in the context of the high-scale SUSY scenario [13-16]. As the wino and gluino are heavier, the unification scale becomes lower, and it can reach the discovery range of $\mathrm{HK}$ for $\sim 10-100 \mathrm{TeV}$ wino and gluino masses.

What about the dimension-6 proton decay in SUSY SO(10) GUTs? The SO(10) GUT is attractive since all the observed fermions (including right-handed neutrinos) can be unified in one spinor representation in each generation. The breaking pattern of the $\mathrm{SO}(10)$ symmetry, however, has room for the existence of intermediate scales, and thus the prediction of the dimension- 6 proton decay varies in a wide range. Among various choices of the Higgs representations to break $\mathrm{SO}(10)$ to the SM gauge symmetry, the simplest choice is $\mathbf{4 5} \mathrm{H}+\mathbf{1 6} \mathbf{H}_{\mathrm{H}}+\overline{\mathbf{1 6}}_{\mathrm{H}}$, which is also the most economical in view of the total contribution to the beta coefficient for gauge couplings. The above choice of the Higgs representations gives characteristic vacua where the GUT gauge boson with SM charge $(\mathbf{3}, \mathbf{2}, 1 / 6)$, which is absent in $\mathrm{SU}(5) \mathrm{GUT}$, is about 1/5 lighter than the GUT gauge boson with SM charge $(\mathbf{3}, \mathbf{2},-5 / 6)$, which is also present in SU(5) GUT. In the vacua, therefore, the dimension- 6 proton decay width is enlarged compared to the minimal SU(5) model due to the exchange of the light $(\mathbf{3}, \mathbf{2}, 1 / 6)$ gauge boson. So, it is worth scrutinizing the prediction of the dimension- 6 proton decay in the above model, since the predicted proton lifetime can be in the range of HK. To our best knowledge, this simple $\mathrm{SO}(10)$ model has not been investigated in light of experimental accessibility of the dimension- 6 proton decay. In this paper, we will show a numerical calculation of the dimension- 6 proton decay $p \rightarrow \pi^{0} e^{+}$in the $\mathrm{SO}(10)$ model with $\mathbf{4 5}_{\mathrm{H}}+\mathbf{1 6}_{\mathrm{H}}+\overline{\mathbf{1 6}}_{\mathrm{H}}$ GUT-breaking Higgs fields.

We also find that in the characteristic vacua of the above model, the colored Higgs mass is enhanced by about 576 compared to the minimal SU(5) model due to threshold corrections of GUT gauge bosons and physical components of GUT-breaking Higgs fields. ${ }^{2}$ So, this $\mathrm{SO}(10)$ model exhibits an interesting tendency that the dimension- 6 decay width is enhanced and the dimension-5 decay width is suppressed.

This paper is organized as follows: in section 2, we present the spectrum of the $\mathrm{SO}(10)$ gauge bosons that gain mass via symmetry breaking with $\mathbf{4 5}_{\mathrm{H}}+\mathbf{1 6}_{\mathrm{H}}+\overline{\mathbf{1 6}}_{\mathrm{H}}$, and show that the dimension- 6 proton decay width can be enlarged. In section 3 , we study how the characteristic $\mathrm{SO}(10)$ breaking vacua of the model are obtained. In section 4, GUTscale threshold corrections are evaluated for the calculation of the dimension- 6 proton decay width. In section 5 , a detailed numerical result for the proton lifetime is presented. Section 6 is for the conclusion. In appendix A, we show the mass spectrum of the multiplets which

\footnotetext{
${ }^{2}$ Considering this enhancement and uncertainty of the Yukawa coupling unification, we omit an analysis of the dimension-5 decay in this paper.
} 
come from $\mathbf{1 6}_{\mathrm{H}}+\overline{\mathbf{1 6}}_{\mathrm{H}}$. In appendix $\mathrm{B}$, an alternative, renormalizable superpotential with $\mathbf{5 4 _ { \mathrm { H } }}$ representation GUT Higgs field is shown.

\section{Spectrum of the $\mathrm{SO}(10)$ gauge bosons and proton decay}

There are many ways to break the $\mathrm{SO}(10)$ gauge symmetry to the SM gauge symmetry. The most economical choice of the Higgs representations to break $\mathrm{SO}(10)$ is $\mathbf{4 5}_{\mathrm{H}}+\mathbf{1 6}_{\mathrm{H}}$. We also introduce $\overline{\mathbf{1 6}}_{\mathrm{H}}$ for $D$-flatness. $\mathbf{4 5} \mathrm{H}$ contains two SM singlets: a SU(5) singlet $\left(a_{1}\right)$ and a SM singlet in SU(5) adjoint $\left(a_{24}\right)$. By general vacuum expectation values (VEVs) of $a_{1}$ and $a_{24}$ (without particular relations between them), $\mathrm{SO}(10)$ is broken down to $\mathrm{SU}(3)_{c} \times \mathrm{SU}(2)_{L} \times \mathrm{U}(1)_{Y} \times \mathrm{U}(1)^{\prime}$. The $\mathrm{SM}$ singlet in $\mathbf{1 6}_{\mathrm{H}}\left(v_{R}\right)$, which is a $\mathrm{SU}(5)$ singlet, develops a VEV to break $\mathrm{U}(1)^{\prime}$ (the singlet in $\overline{\mathbf{1 6}}_{\mathrm{H}}\left(\bar{v}_{R}\right)$ also gains a VEV with $\left|v_{R}\right|=\left|\bar{v}_{R}\right|$ to keep D-flatness). Due to the absence of cubic term of $\mathbf{4 5} \mathbf{H}_{\mathrm{H}}, a_{24}$ cannot acquire a VEV from the renormalizable superpotential. However, by introducing non-renormalizable, quartic terms of $\mathbf{4 5} \mathbf{5}_{\mathrm{H}}, a_{24}$ can acquire a VEV to break $\mathrm{SU}(5)$.

The GUT gauge boson masses generated by the VEVs of $\mathbf{4 5} \mathbf{H}_{\mathrm{H}}, \mathbf{1 6}_{\mathrm{H}}$ and $\overline{\mathbf{1 6}}_{\mathrm{H}}$ are

$$
\begin{aligned}
& M_{X}^{2} / g_{5}^{2}=\frac{5}{6}\left|a_{24}\right|^{2}, \\
& M_{Q}^{2} / g_{5}^{2}=\frac{4}{5}\left|a_{1}+\frac{1}{2 \sqrt{6}} a_{24}\right|^{2}+\left|v_{R}\right|^{2}, \\
& M_{U}^{2} / g_{5}^{2}=\frac{4}{5}\left|a_{1}-\sqrt{\frac{2}{3}} a_{24}\right|^{2}+\left|v_{R}\right|^{2}, \\
& M_{E}^{2} / g_{5}^{2}=\frac{4}{5}\left|a_{1}+\sqrt{\frac{3}{2}} a_{24}\right|^{2}+\left|v_{R}\right|^{2},
\end{aligned}
$$

where $X, Q, U$ and $E$ denote $\mathrm{SO}(10)$ gauge bosons whose SM charges are $X:(\mathbf{3}, \mathbf{2},-5 / 6)$, $Q:(\mathbf{3}, \mathbf{2}, 1 / 6), U:(\mathbf{3}, \mathbf{1}, 2 / 3)$ and $E:(\mathbf{1}, \mathbf{1}, 1)$, and $a_{1}, a_{24}$ and $v_{R}$ are the VEVs of canonically-normalized SM singlets. The extra U(1) gauge boson mass is $g_{5} \sqrt{5 / 2} v_{R}$.

When $a_{24} \gg a_{1}, v_{R}$, we obtain

$$
M_{X}: M_{Q}: M_{U}: M_{E} \simeq 5: 1: 4: 6 .
$$

This ratio is easily obtained by the rule $(3+2):(3-2):(2+2):(3+3)$.

The dimension- 6 proton decay operators are generated not only by the $X$ gauge boson exchange but also by the $Q$ gauge boson exchange. The partial width of the dimension- 6 proton decay is given by

$$
\Gamma \propto A_{R}^{2}\left(\frac{1}{M_{X}^{2}}+\frac{1}{M_{Q}^{2}}\right)^{2}+\frac{4 A_{L}^{2}}{M_{X}^{4}},
$$

where $A_{L, R}$ are the renormalization factors for $q \ell\left(u^{c}\right)^{\dagger}\left(d^{c}\right)^{\dagger}$ and $q q\left(e^{c}\right)^{\dagger}\left(u^{c}\right)^{\dagger}$ operators. One finds that the $Q$ gauge boson exchange gives much larger contribution when $M_{X}: M_{Q} \simeq$ 
$5: 1$. The ratio of the decay width in $\mathrm{SU}(5)$ GUT $\left(M_{Q} \rightarrow \infty\right)$ and in the $\mathrm{SO}(10)$ GUT with $a_{24} \gg a_{1}, v_{R}$ (now) is

$$
\Gamma^{\mathrm{SU}(5)}: \Gamma^{\mathrm{now}} \simeq 1: 136
$$

for $A_{L} \simeq A_{R}$, if the $X$ gauge boson masses are the same. Since the naive prediction of $p \rightarrow \pi^{0} e^{+}$partial lifetime in SU(5) GUT is $\tau_{p} \sim 10^{36}$ years, the prediction in the $\mathrm{SO}(10)$ with $a_{24} \gg a_{1}, v_{R}$ is $10^{34}$ years, which is on the current experimental bound at SK.

\section{$3 \mathrm{SO}(10)$ breaking vacua in the model}

We consider a superpotential for the GUT breaking Higgs fields $\mathbf{4 5}_{\mathrm{H}}(A), \mathbf{1 6}_{\mathrm{H}}(\chi)$ and $\overline{\mathbf{1 6}}_{\mathrm{H}}$ $(\bar{\chi})$

$$
W=\frac{1}{2} m_{A} A^{2}+\frac{\lambda_{1}}{M_{*}}\left(A^{2}\right)^{2}+\frac{\lambda_{2}}{M_{*}} A^{4}+m_{\chi} \chi \bar{\chi}+\kappa A \chi \bar{\chi}
$$

where we define $A^{2} \equiv A_{a b} A_{a b} / 2$, and $A^{4} \equiv A_{a c} A_{a d} A_{b c} A_{b d} / 2$ so that the multiplication of contraction of 2-anti-symmetric indices is removed by dividing by 2 . The superpotential in terms of canonically-normalized SM singlets $a_{1}, a_{24}$ in $A$ and $v_{R}$ in $\chi$ (Clebsch-Gordan coefficients for $\mathbf{1 6}$ representation can be found in [18]) is given by

$$
\begin{aligned}
\left.W\right|_{\text {SM singlets }}= & m_{\chi} v_{R} \bar{v}_{R}+\frac{1}{2} m_{A}\left(a_{1}^{2}+a_{24}^{2}\right)+\sqrt{5} \kappa a_{1} v_{R} \bar{v}_{R} \\
& +\frac{\lambda_{1}}{M_{*}}\left(a_{1}^{2}+a_{24}^{2}\right)^{2}+\frac{\lambda_{2}}{5 M_{*}}\left(a_{1}^{4}+6 a_{1}^{2} a_{24}^{2}+2 \sqrt{\frac{2}{3}} a_{1} a_{24}^{3}+\frac{7}{6} a_{24}^{4}\right) .
\end{aligned}
$$

The $F$-flat conditions read

$$
\begin{aligned}
& a_{1}=-\frac{m_{\chi}}{\sqrt{5} \kappa}, \\
& a_{24}=\frac{m_{\chi}}{\sqrt{5} \kappa} a, \\
& v_{R} \bar{v}_{R}=\frac{4 m_{\chi}^{3}}{25 \kappa^{4} M_{*}}\left(\lambda_{1}\left(1+a^{2}\right)+\frac{\lambda_{2}}{5}\left(1+3 a^{2}-\frac{1}{\sqrt{6}} a^{3}\right)\right)+\frac{m_{\chi} m_{A}}{5 \kappa^{2}},
\end{aligned}
$$

where $a$ is a solution to

$$
\lambda_{1}+\frac{3}{5} \lambda_{2}+\frac{5 \kappa^{2} m_{A} M_{*}}{4 m_{\chi}^{2}}-\sqrt{\frac{3}{2}} \frac{\lambda_{2}}{5} a+\left(\lambda_{1}+\frac{7}{30} \lambda_{2}\right) a^{2}=0 .
$$

In eq. (3.3), the condition $\partial W / \partial v_{R}=\left(m_{\chi}+\sqrt{5} \kappa a_{1}\right) \bar{v}_{R}$ fixes the VEV of $a_{1}$ to be around $m_{\chi}$. In eq. (3.6), on the other hand, the VEV of $a_{24}$ (proportional to $a$ ) is fixed by a balance between the quadratic mass term and the quartic non-renormalizable term, and $|a|$ is large if $\left|m_{A} M_{*} / m_{\chi}^{2}\right| \gg 1$, in which case we obtain

$$
a_{24}^{2} \sim-\frac{m_{A} M_{*}}{4\left(\lambda_{1}+\frac{7}{30} \lambda_{2}\right)}, \quad v_{R} \bar{v}_{R} \sim \frac{\lambda_{2}}{5 \sqrt{30} \kappa} \frac{m_{A} a_{24}}{\lambda_{1}+\frac{7}{30} \lambda_{2}} .
$$

Thus, vacua with $\left|v_{R}\right|,\left|a_{1}\right| \ll\left|a_{24}\right|$ are obtained $^{3}$ with a feasible assumption $m_{\chi}, m_{A} \ll M_{*}$.

\footnotetext{
${ }^{3}$ If one adds a non-renormalizable term $(\chi \bar{\chi})^{2} / M_{*}$ to the superpotential, new vacua with $v_{R} \sim \sqrt{m_{\chi} M_{*}}$ appear. However, the vacua we obtain in the main text remain stable with a correction of $O\left(v_{R}^{2} / M_{*}^{2}\right)$.
} 


\section{GUT-scale threshold corrections for the gauge coupling unification}

The gauge coupling unification conditions [25] in SUSY SO(10) GUT are written as ${ }^{4}$

$$
\begin{aligned}
-\frac{2}{\alpha_{3}\left(m_{Z}\right)}+\frac{3}{\alpha_{2}\left(m_{Z}\right)}-\frac{1}{\alpha_{1}\left(m_{Z}\right)} & =\frac{1}{2 \pi}\left(\frac{12}{5} \ln \frac{M_{H}}{m_{Z}}-2 \ln \frac{m_{\mathrm{SUSY} 1}}{m_{Z}}\right), \\
-\frac{2}{\alpha_{3}\left(m_{Z}\right)}-\frac{3}{\alpha_{2}\left(m_{Z}\right)}+\frac{5}{\alpha_{1}\left(m_{Z}\right)} & =\frac{1}{2 \pi}\left(36 \ln \frac{M_{G}}{m_{Z}}+8 \ln \frac{m_{\mathrm{SUSY} 2}}{m_{Z}}\right), \\
M_{H} & =\frac{M_{Q}^{4}}{M_{E} M_{U}^{3}} M_{H_{C}} \prod_{i} M_{i}^{l_{A}^{i}} \\
M_{G}^{6} & =\frac{M_{X}^{4} M_{E}^{2} M_{U}^{2}}{M_{Q}^{4}} \prod_{i} M_{i}^{l_{B}^{i}},
\end{aligned}
$$

where $M_{X, Q, U, E}$ are the $\mathrm{SO}(10)$ gauge boson masses which we have already defined, $M_{H_{C}}$ is the colored Higgs mass, and $i$ stands for the degree of physical modes under the SM decompositions. We define $l_{A}=\frac{5}{12}\left(2 l_{3}-3 l_{2}+l_{1}\right)$ and $l_{B}=\frac{1}{6}\left(2 l_{3}+3 l_{2}-5 l_{1}\right)$ where $l_{i}$ gives the beta coefficient contribution of the respective multiplet, $l_{i}=\Delta b_{i}^{\text {SUSY }}$. Because the would-beGoldstone modes which are eaten by the gauge bosons lack from the multiplets, we obtain

$$
\sum_{i} l_{A}^{i}=0, \quad \sum_{i} l_{B}^{i}=2 .
$$

The RGEs give

$$
M_{H} \sim 10^{15}-10^{16} \mathrm{GeV}, \quad M_{G} \sim 2 \times 10^{16} \mathrm{GeV},
$$

and the GUT gauge boson and colored Higgs masses depend on threshold corrections of GUT-scale particles.

The $\mathbf{4 5 _ { \mathrm { H } }}$ and $\mathbf{1 6} \mathbf{6}_{\mathrm{H}}$ representations are decomposed under $\mathrm{SU}(5)$ as $\mathbf{4 5} \mathbf{5}_{\mathrm{H}}=\mathbf{2 4}+\mathbf{1 0}+$ $\overline{\mathbf{1 0}}+\mathbf{1}$. and $1 \mathbf{6}_{\mathrm{H}}=\mathbf{1 0}+\overline{\mathbf{5}}+\mathbf{1}$. One linear combination of the 10's (and $\overline{\mathbf{1 0}}$ 's) from $\mathbf{4 5}_{\mathrm{H}}$ and $\mathbf{1 6} \mathbf{H}_{\mathrm{H}}\left(\overline{\mathbf{1 6}}_{\mathrm{H}}\right)$ is absorbed by the gauge bosons $Q, U, E$. For $\left|v_{R}\right| \ll\left|a_{24}\right|$, the linear combination to be absorbed mainly comes from $\mathbf{4 5} \mathbf{5}_{\mathrm{H}}$. The other linear combination is a physical mode and we denote its components by $\chi_{Q}, \chi_{U}, \chi_{E}$ (which respectively have the same SM charge as $Q, U, E)$. For $\left|m_{A}\right|,\left|m_{\chi}\right| \ll M_{*}$, their masses satisfy the ratio (see appendix A for the derivation)

$$
M_{\chi_{Q}}: M_{\chi_{U}}: M_{\chi_{E}} \simeq 1: 4: 6 .
$$

${ }^{4}$ The SUSY particle threshold contributions in the respective equations are more precisely written as

$$
\begin{aligned}
-2 \ln \frac{m_{\mathrm{SUSY} 1}}{m_{Z}} & =-\frac{8}{5} \ln \frac{\mu_{H}}{m_{Z}}-\frac{2}{5} \ln \frac{m_{H}}{m_{Z}}+4 \ln \frac{M_{\tilde{g}}}{M_{\tilde{w}}}+\frac{3}{5} \ln \frac{m_{\tilde{q}^{c}}^{3} m_{\tilde{d}^{c}}^{2} m_{\tilde{e}^{c}}}{m_{\tilde{q}}^{4} m_{\tilde{\ell}}^{2}}, \\
8 \ln \frac{m_{\mathrm{SUSY} 2}}{m_{Z}} & =4 \ln \frac{M_{\tilde{g}} M_{\tilde{w}}}{m_{Z}^{2}}+3 \ln \frac{m_{\tilde{q}}^{2}}{m_{\tilde{u}} m_{\tilde{e}^{c}}}
\end{aligned}
$$

where $\mu_{H}, m_{H}$ are higgsino and heavier Higgs masses, $M_{\tilde{g}}$ and $M_{\tilde{w}}$ are gluino and wino masses. From these equations, one finds that the colored Higgs mass is larger for a smaller ratio of $M_{\tilde{g}} / M_{\tilde{w}}$, and the unification scale $M_{G}$ becomes smaller for heavier wino and gluino masses. 
The $\mathbf{2 4}$ representation in $\mathbf{4 5} \mathbf{H}_{\mathrm{H}}$ contains a $\mathrm{SU}(3)_{c}$ adjoint $(\mathbf{8}, \mathbf{1}, 0)$ and a $\mathrm{SU}(2)_{L}$ adjoint $(\mathbf{1}, \mathbf{3}, 0)$ as physical modes. Their masses can be calculated (using the minimization conditions) as

$$
\begin{aligned}
M_{8} & =\frac{2}{3} \frac{\lambda_{2}}{M_{*}} a_{24}\left(-3 \sqrt{6} a_{1}+a_{24}\right), \\
M_{3} & =\frac{2}{3} \frac{\lambda_{2}}{M_{*}} a_{24}\left(3 \sqrt{6} a_{1}+4 a_{24}\right),
\end{aligned}
$$

and when $\left|a_{1}\right| \ll\left|a_{24}\right|$, we find

$$
M_{8}: M_{3} \simeq 1: 4 \text {. }
$$

The other physical Higgs modes are the $\mathbf{5}+\overline{\mathbf{5}}$ in $\mathbf{1 6}_{\mathrm{H}}+\overline{\mathbf{1 6}}_{\mathrm{H}}$. We additionally introduce two $10_{\mathrm{H}}$ representations to generate renormalizable Yukawa couplings that give fermion masses after electroweak symmetry breaking. Then, there are three heavy colored Higgs fields $H_{C}, H_{T_{1,2}}$, and two heavy Higgs doublets $H_{D_{1,2}}$ around the GUT scale.

Now the gauge coupling unification conditions are specified as

$$
\begin{aligned}
M_{H} & =\frac{M_{Q}^{4}}{M_{E} M_{U}^{3}} \frac{\left(M_{\chi_{E}} M_{\chi_{U}}^{3}\right)^{1 / 2}}{M_{\chi_{Q}}^{2}}\left(\frac{M_{8}}{M_{3}}\right)^{5 / 2} \frac{M_{T_{1}} M_{T_{2}}}{M_{D_{1}} M_{D_{2}}} M_{H_{C}} \\
M_{G}^{6} & =\frac{M_{E}^{2} M_{U}^{2}}{M_{Q}^{4}} \frac{M_{\chi_{Q}}^{2}}{M_{\chi_{E}} M_{\chi_{U}}} M_{X}^{4} M_{8} M_{3} .
\end{aligned}
$$

In the vacua with $\left|v_{R}\right|,\left|a_{1}\right| \ll\left|a_{24}\right|$, we obtain from eqs. (2.5), (4.9), (4.12),

$$
\begin{aligned}
\frac{M_{Q}^{4}}{M_{E} M_{U}^{3}} \frac{\left(M_{\chi_{E}} M_{\chi_{U}}^{3}\right)^{1 / 2}}{M_{\chi_{Q}}^{2}}\left(\frac{M_{8}}{M_{3}}\right)^{5 / 2} & \simeq \frac{1}{576}, \\
\frac{M_{E}^{2} M_{U}^{2}}{M_{Q}^{4}} \frac{M_{\chi_{Q}}^{2}}{M_{\chi_{E}} M_{\chi_{U}}} & \simeq 24 .
\end{aligned}
$$

Due to the factor $1 / 576$, the colored Higgs mass can be much larger than in the minimal $\mathrm{SU}(5)$ model. As for the gauge boson mass, in the minimal SU(5) model, one has $M_{G}^{6}=$ $M_{X}^{4} M_{8} M_{3}$ and $M_{8}=M_{3}=\lambda M_{X}$ where $\lambda$ is proportional to the self-coupling of the $\mathrm{SU}(5)$ adjoint representation. $\lambda$ is arbitrary unless it far exceeds $O(1)$, and people often assume $\lambda \sim 1$, which gives $M_{X} \sim M_{G}$. In the current $\operatorname{SO}(10)$-breaking vacua $\left|v_{R}\right|,\left|a_{1}\right| \ll\left|a_{24}\right|$, if we write $4 M_{8} \simeq M_{3}=\rho M_{X}, \rho$ is always much smaller than 1 because the masses of the $\mathrm{SU}(3)_{c}$ adjoint and $\mathrm{SU}(2)_{L}$ adjoint particles are roughly $m_{A}$, while the $X$ gauge boson mass is roughly $\left(m_{A} M_{*}\right)^{1 / 2}$. To be specific, we get from eqs. (4.14), (4.16),

$$
M_{X} \simeq M_{G} \frac{1}{\left(6 \rho^{2}\right)^{1 / 6}}
$$

and from eqs. (2.1), (4.11),

$$
\rho=\frac{M_{3}}{M_{X}} \simeq \frac{16}{5} \lambda_{2} \frac{1}{g_{5}^{2}} \frac{M_{X}}{M_{*}}
$$

Therefore we find

$$
\rho \simeq 2\left(\frac{\lambda_{2}}{g_{5}^{2}}\right)^{3 / 4}\left(\frac{M_{G}}{M_{*}}\right)^{3 / 4}
$$


which equals 0.1 for $\lambda_{2}=1$ and $M_{*}=2 \times 10^{18} \mathrm{GeV}$. It follows that $M_{X}$ is a little larger than $M_{G}$. Nevertheless, the $Q$ gauge boson satisfying $M_{X}: M_{Q} \simeq 5: 1$ enhances the dimension-6 proton decay width compared to the minimal SU(5) model.

To summarize, in the $\mathrm{SO}(10)$-breaking vacua with $\left|a_{24}\right| \gg\left|a_{1}\right|,\left|v_{R}\right|$, the colored Higgs is made heavier by the GUT-scale threshold corrections, and the dimension- 5 proton decay is suppressed compared to the minimal SU(5) model. On the other hand, the dimension- 6 proton decay width is roughly 100 times enlarged and we have $\tau_{p} \sim 10^{34}$ years, which is in the scope of HK.

Suppression of the dimension-5 proton decay is also achieved by making the ratio of gluino and wino masses $M_{\tilde{g}} / M_{\tilde{w}}$ smaller, and enhancement of the dimension-6 proton decay is achieved by increasing their product $M_{\tilde{g}} M_{\tilde{w}}$, as seen from the SUSY particle threshold correction formulas. Hence, in the high scale SUSY scenario, the dimension-6 proton decay is detectable at HK even in the minimal SUSY SU(5) model. In contrast, in our $\mathrm{SO}(10)$-breaking vacua $\left|a_{24}\right| \gg\left|a_{1}\right|,\left|v_{R}\right|$, the GUT-scale threshold corrections enhance the dimension-6 proton decay width to a detectable level, even if SUSY particle masses are a few $\mathrm{TeV}$.

We comment on the case when the $\mathbf{1 6} \mathbf{H}_{\mathrm{H}}$ is replaced by $\mathbf{1 2 6}_{\mathrm{H}}$ representation. Choice of $\mathbf{1 6}_{H}$ needs additional $R$-parity symmetry to avoid rapid dimension-4 proton decays, and adopting $\mathbf{1 2 6}_{H}$ has a merit that $R$-parity in MSSM is induced due to the fact that the vevs of $\mathbf{1 2 6}_{H}, \overline{\mathbf{1 2 6}}_{H}$ have $B-L= \pm 2$ (while the vevs of $\mathbf{1 6}_{H}, \overline{\mathbf{1 6}}_{H}$ have $B-L= \pm 1$ ). However, when a vacuum with $\left|a_{1}\right| \ll\left|a_{24}\right|$ is chosen, $(\mathbf{6}, \mathbf{3}, 1 / 3)$ multiplet in $\mathbf{1 2 6}_{H}$ is about $1 / 3$ lighter than the other components in the representation. Since this multiplet has $l_{A}=-33 / 2$, it gives a large threshold correction and renders the colored Higgs too light. To avoid this unwanted large thresholds, we adopt the choice of $\mathbf{1 6}_{H}$ (with $R$-parity) in this paper.

\section{Numerical result}

In the previous section, we have used 1-loop relations to describe qualitative behaviors. In this section, we will show a numerical result using 2-loop RGE evolutions [19, 20]. In the result, we use the central value of the 5-flavor strong coupling, $\alpha_{s}^{(5)}\left(M_{Z}\right)^{\overline{\mathrm{MS}}}=$ $0.1181 \pm 0.0011$ [21]. The colored Higgs mass is sensitive to the value of the strong coupling, while the GUT gauge boson masses are less sensitive. The proton lifetime is about $50 \%$ larger if we use the value $+3 \sigma$. We assume all the SUSY particle masses to be $2 \mathrm{TeV}$ except for the wino mass, which is taken to be $500 \mathrm{GeV}$.

The decay width of $p \rightarrow \pi^{0} e^{+}$is [22]

$$
\Gamma=\frac{\pi}{4} \frac{m_{p} \alpha_{H}^{2}}{f_{\pi}^{2}}(1+F+D)^{2} \alpha_{U}^{2}\left[A_{R}^{2}\left(\frac{1}{M_{X}^{2}}+\frac{1}{M_{Q}^{2}}\right)^{2}+\frac{4 A_{L}^{2}}{M_{X}^{4}}\right],
$$

where we use proton mass $m_{p}=0.9383 \mathrm{GeV}$, chiral Lagrangian parameters $F=0.46$, $D=0.80$, hadron matrix element for proton decay $\alpha_{H}=-0.014 \mathrm{GeV}^{3}$ at $2 \mathrm{GeV}$ [23], decay constant $f_{\pi}=0.1307 \mathrm{GeV}$, renormalization factors down to $2 \mathrm{GeV}, A_{L}=2.91$, $A_{R}=2.78$ (The two-loop renormalization factors are calculated in [24]). From the $\mathrm{SO}(10)$ gauge coupling unification, we obtain $1 / \alpha_{U}\left(M_{X}\right)=4 \pi / g_{5}^{2} \simeq 25.2$. 
Before presenting the main result, we show an estimate on the partial proton lifetime. Under the approximations with

$$
\begin{aligned}
M_{X}: M_{3}: M_{8} & =1: \rho: \rho / 4, \\
M_{\chi_{Q}}: M_{\chi_{U}}: M_{\chi_{E}} & =1: 4: 6,
\end{aligned}
$$

and

$$
M_{X}: M_{Q}: M_{U}: M_{E}=5: 1: 4: 6
$$

the partial proton lifetime is found to be

$$
\tau_{p} \simeq\left(\frac{0.1}{\rho}\right)^{\frac{4}{3}} \times 2.6 \times 10^{34} \text { years. }
$$

As discussed in the previous section, $\rho \ll 1$ in the current $\mathrm{SO}(10)$-breaking vacua because the VEV of $a_{24}$ is roughly the geometrical average of $m_{A}$ and $M_{*}$ while $M_{3}, M_{8}$ are roughly $m_{A}$, and we get $\rho \simeq 0.1$ for $\lambda_{2}=1$ and $M_{*}=2 \times 10^{18} \mathrm{GeV}$.

It is interesting to compare the above estimate with the prediction of the minimal $\mathrm{SU}(5)$ model. In the minimal SU(5), we define $M_{8}=M_{3}=\lambda M_{X}$ where $\lambda$ is proportional to the self-coupling of the adjoint field that breaks $\mathrm{SU}(5)$. Then, the partial proton lifetime is found to be $\mathrm{e}^{5}$

$$
\tau_{p}^{\mathrm{SU}(5)} \simeq \lambda^{-4 / 3} \times 5.5 \times 10^{35} \text { years }
$$

We observe that the partial lifetime decreases by $1 / 20$ in our $\mathrm{SO}(10)$-breaking vacua compared to the minimal SU(5) model, for natural values of $\rho=0.1$ and $\lambda=1$.

The estimate for our $\mathrm{SO}(10)$-breaking vacua, eq. (5.5), receives corrections from the small VEVs of $a_{1}, v_{R}$ that perturb the mass ratios. In table 1 , we show precise numerical values. Here, we fix $M_{*}=2 \times 10^{18} \mathrm{GeV}$, and take benchmark values for $\lambda_{1}, \lambda_{2}, \kappa$ and $m_{\chi}$. We solve the $F$-flat conditions eqs. (3.3)-(3.6) and the unification conditions by varying $m_{A}$ and the colored Higgs mass. Since eq. (3.6) is a quadratic equation, there are two solutions. If $m_{A}<0, a_{24}$ is real and the mass spectrum splits into two, both of which are tabulated. If $m_{A}>0, a_{24}$ is complex and the two solutions yield the same mass spectrum in terms of the absolute values. From (iii) and (iii") of table 1, we find that the mass spectrum is not sensitive to $\lambda_{1}$. This is because the relation $\left|a_{1}\right|,\left|v_{R}\right| \ll\left|a_{24}\right|$ gives $M_{3} / a_{24} \propto \lambda_{2} a_{24} / M_{*}$. Although $a_{24}$ depends on $\lambda_{1}$, the ratio $M_{3} / M_{X}$ does not depend on $\lambda_{1}$ for $\left|a_{1}\right|,\left|v_{R}\right| \ll\left|a_{24}\right|$. As a result, once $M_{X}$ is chosen to realize the gauge coupling unification, the mass spectrum is almost independent of $\lambda_{1}$. On the other hand, when $\lambda_{2}$ is smaller, the $\mathrm{SU}(3)_{c}$ adjoint and $\mathrm{SU}(2)_{L}$ adjoint particles become lighter ( $\rho=M_{3} / M_{X}$ is smaller), and the proton lifetime becomes longer, as seen from (iii) and (iii') of table 1. Consequently, the proton lifetime cannot be bounded from above theoretically. Still, it is interesting that for $\lambda_{2} \sim 1$, the dimension-6 proton decay is detectable at HK.

In the benchmarks of table 1 , the effective colored Higgs mass, $M_{H_{T}}=\frac{M_{T_{1}} M_{T_{2}} M_{H_{C}}}{M_{D_{1}} M_{D_{2}}}$, is $2 \times 10^{17} \mathrm{GeV}$. The relation $M_{H_{T}}>M_{X}$ is realized with a large coupling of $A H_{1} H_{2}$ (see

\footnotetext{
${ }^{5}$ We remark that the LHC bound of gluino mass reduces the dimension- 6 partial lifetime due to eqs. (4.2) and (4.4), though the prediction of the minimal SUSY SU(5) model is often referred to be $10^{36}$ years.
} 


\begin{tabular}{|c|c|c|c|c|c|c|c|c|c|}
\hline & $\left(\lambda_{1}, \lambda_{2}, \kappa\right)$ & $m_{\chi}$ & $m_{A}$ & $M_{X}$ & $M_{Q}$ & $M_{3}$ & $M_{8}$ & $v_{R}$ & $\tau_{p}\left(10^{34}\right.$ years $)$ \\
\hline (i-A) & $(1,1,1)$ & 0.1 & -0.47 & 2.8 & 0.55 & 0.25 & 0.068 & 0.24 & 3.4 \\
\hline (i-B) & $(1,1,1)$ & 0.1 & -0.46 & 2.8 & 0.61 & 0.25 & 0.057 & 0.25 & 4.7 \\
\hline (ii-A) & $(1,1,1)$ & 0.01 & -0.48 & 2.8 & 0.59 & 0.26 & 0.069 & 0.25 & 4.3 \\
\hline (ii-B) $^{\text {iii) }}$ & $(1,1,1)$ & 0.01 & -0.44 & 2.7 & 0.57 & 0.24 & 0.059 & 0.24 & 3.7 \\
\hline (iii) $^{(1,1,1)}$ & 0.1 & 0.46 & 2.8 & 0.58 & 0.25 & 0.063 & 0.24 & 4.0 \\
\hline (iii $\left.^{\prime}\right)$ & $(1,0.5,1)$ & 0.1 & 0.58 & 3.2 & 0.67 & 0.17 & 0.043 & 0.22 & 7.2 \\
\hline (iii' $\left.^{\prime \prime}\right)$ & $(0.1,1,1)$ & 0.1 & 0.12 & 2.8 & 0.58 & 0.25 & 0.063 & 0.24 & 4.0 \\
\hline (iii' $\left.^{\prime \prime}\right)$ & $(1,1,0.5)$ & 0.1 & 0.48 & 2.8 & 0.62 & 0.26 & 0.066 & 0.36 & 5.1 \\
\hline
\end{tabular}

Table 1. The $p \rightarrow \pi^{0} e^{+}$partial lifetime and the mass spectrum for various input values of $\lambda_{1}, \lambda_{2}, \kappa$ and $m_{\chi}$. The masses and VEV are in units of $10^{16} \mathrm{GeV}$. From (i-A) to (ii-B), $m_{A}<0$ and thus $a_{24}$ is real and the mass spectrum splits into two, for which (i-A) and (ii-A) correspond to the cases with $a_{24}>0$, and (i-B) and (ii-B) to the cases with $a_{24}<0$. In (iii), $m_{A}>0$ and thus $a_{24}$ is complex. We change one of $\lambda_{1}, \lambda_{2}, \kappa$ in ( $\left.\mathrm{iii}^{\prime}\right),\left(\mathrm{iii}^{\prime \prime}\right)$, ( $\left.\mathrm{iii}^{\prime \prime \prime}\right)$.

appendix A). Since the dimension-5 proton decay amplitudes also depend on details of the Yukawa coupling unification, we do not discuss the dimension-5 decay in this paper.

Before concluding, we comment on the realization of fermion masses and mixings in the model. All the quarks and leptons including the right-handed neutrinos are unified in spinor representation $\psi_{i}$ ( $i$ stands for a generation index). The right-handed neutrino masses are generated by $\psi_{i} \psi_{j} \bar{\chi} \bar{\chi}$ (or $S \psi_{i} \bar{\chi}+M_{S} S^{2}$ with singlet field $S$ ). The Yukawa interaction (including Dirac neutrino Yukawa interaction) can come from $Y_{1}^{i j} \psi_{i} \psi_{j} H_{1}+Y_{2}^{i j} \psi_{i} \psi_{j} H_{2}$, where $H_{1}$ and $H_{2}$ are 10-dimensional reps, as well as non-renormalizable terms such as $\psi_{i} \psi_{j} \chi \chi$. The MSSM Higgs doublets are contained in $H_{1}, H_{2}$, and $\chi$ and $\bar{\chi}$, which are mixed and one linear combinations are light (see appendix A). Because of the non-trivial mixings of Higgs doublets, CKM mixings are generated. The Higgs mixings can also induce the freedom of $\tan \beta$, which is the ratio of the Higgs doublet vevs. Suppose that $Y_{1}^{33}$ gives the top Yukawa coupling and up-type Higgs doublet is contained in $H_{1}$ dominantly. In order to break the up-down symmetry and generate CKM mixings, down-type Higgs doublet should contain $H_{2}$ component. In order to realize the muon/strange and electron/down-quark mass ratios, one needs $Y_{2}^{\prime 22} \psi_{2} \psi_{2} A H_{2} / M_{*}$ term. The muon Yukawa coupling is about 0.025 for $\tan \beta \simeq$ 50 and one can find that $Y_{2}^{22} \sim Y_{2}^{\prime 22} 3 / \sqrt{30} a_{24} / M_{*} \sim 0.01$ can realize the muon/strange masses. In the current fit, $a_{24}$ can be calculated from eq. (2.1) and one can check that $Y_{2}^{\prime 22} \simeq 1$ (with $M_{*}=2 \times 10^{18} \mathrm{GeV}$ ) can realize the proper masses. Because of the freedom of Higgs mixing and $\tan \beta$, the coefficient can be even smaller. After all, the fermion masses and mixings (including the neutrino mixings) can be realized in the current setup of $\mathrm{SO}(10)$ model. Compared to the models with $\mathbf{1 2 6}_{H}$, relations of fermion masses and mixings are less predictive, and we do not touch more detail of the Yukawa structure in this paper. 


\section{Conclusion}

We have studied the dimension-6 proton decay in a SUSY SO(10) GUT with only $\mathbf{4 5} \mathbf{H}_{\mathrm{H}}+$ $\mathbf{1 6}_{\mathrm{H}}+\overline{\mathbf{1 6}}_{\mathrm{H}}$ GUT-breaking Higgs fields. Since the SU(5)-breaking VEV of $\mathbf{4 5}_{\mathrm{H}}$ is induced by the Planck-suppressed, quartic superpotential for $\mathbf{4 5} \mathbf{5}_{\mathrm{H}}$, this VEV is larger than the SU(5)conserving VEVs. This results in a $1 / 5$ suppression of the $Q(\mathbf{3}, \mathbf{2}, 1 / 6)$ gauge boson mass compared to the $X(\mathbf{3}, \mathbf{2},-5 / 6)$ gauge boson mass. On the other hand, the masses of the $\mathrm{SU}(3)_{c}$ adjoint and $\mathrm{SU}(2)_{L}$ adjoint particles from $\mathbf{4 5} 5_{\mathrm{H}}$ are much smaller than the $X$ gauge boson mass and this enhances the latter when the unification condition is fulfilled. Still, the mass of the $Q$ gauge boson can be below $0.6 \times 10^{16} \mathrm{GeV}$ (for $\lambda_{2}=1$ and $M_{*}=2 \times 10^{18} \mathrm{GeV}$ ) and can thus give rise to a fast dimension- 6 proton decay detectable at Hyper-Kamiokande.

\section{Acknowledgments}

This work is partially supported by Scientific Grants by the Ministry of Education, Culture, Sports, Science and Technology of Japan, Nos. 17K05415, 18H04590 and 19H051061 (NH), and No. 19K147101 (TY).

\section{A Mass spectrum}

$\mathbf{4 5} \mathbf{H}_{\mathrm{H}}(A)$ and $\mathbf{1 6}_{\mathrm{H}}(\chi)$ representations are decomposed under $\mathrm{SU}(5)$ as $\mathbf{4 5 _ { \mathrm { H } }}=\mathbf{2 4}+\mathbf{1 0}+\overline{\mathbf{1 0}}+\mathbf{1}$ and $16_{\mathrm{H}}=\mathbf{1 0}+\overline{\mathbf{5}}+\mathbf{1}$. One linear combination of the 10's (and $\overline{\mathbf{1 0}}$ 's) from $\mathbf{4 5} \mathbf{H}_{\mathrm{H}}$ and $\mathbf{1 6}_{\mathrm{H}}$ $\left(\overline{\mathbf{1 6}}_{\mathrm{H}}\right)$ is absorbed by GUT gauge bosons, $Q:(\mathbf{3}, \mathbf{2}, 1 / 6), U:(\overline{\mathbf{3}}, \mathbf{1},-2 / 3)$, and $E:(\mathbf{1}, \mathbf{1}, 1)$. The other linear combination yields physical modes $\chi_{Q}:(\mathbf{3}, \mathbf{2}, 1 / 6), \chi_{U}:(\overline{\mathbf{3}}, \mathbf{1},-2 / 3)$, and $\chi_{E}:(\mathbf{1}, \mathbf{1}, 1)$. The mass matrix of each component of the $\mathbf{1 0}+\overline{\mathbf{1 0}}$ 's can be written as

$$
\left(\begin{array}{cc}
M_{A A} & M_{A \bar{\chi}} \\
M_{\chi A} & M_{\chi \bar{\chi}}
\end{array}\right),
$$

where

$$
\begin{aligned}
& M_{A A}=m_{A}+4 a_{1}^{2} \frac{\lambda_{1}+\frac{1}{5} \lambda_{2}}{M_{*}}+4 a_{24}^{2} \frac{\lambda_{1}}{M_{*}}+\frac{4}{5} \frac{\lambda_{2}}{M_{*}}\left(\frac{C_{Q, U, E}}{\sqrt{6}} a_{1} a_{24}+\frac{D_{Q, U, E}}{6} a_{24}^{2}\right), \\
& M_{A \bar{\chi}}=2 \kappa v_{R}, \\
& M_{\chi A}=2 \kappa \bar{v}_{R}, \\
& M_{\chi \bar{\chi}}=m_{\chi}+\frac{1}{\sqrt{5}} \kappa a_{1}-\sqrt{\frac{2}{15}} \kappa C_{Q, U, E} a_{24}
\end{aligned}
$$

where $\left(C_{Q}, C_{U}, C_{E}\right)=(1,-4,6)$ and $\left(D_{Q}, D_{U}, D_{E}\right)=(19,4,9)$. We can verify that one eigenvalue is zero when the $F$-flat conditions are used. The mass of the physical mode is $M_{A A}+M_{\chi \bar{\chi}}$. In the limit with $m_{A}, m_{\chi} \ll M_{*}, M_{\chi \bar{\chi}}$ dominates, but $M_{A A}$ can be nonnegligible for $\chi_{Q}$ due to the large factor $D_{Q} / C_{Q}$. Using the minimization condition, we obtain $M_{A A} \simeq m_{A}\left(7-D_{Q, U, E}\right) \lambda_{2} /\left(30 \lambda_{1}+7 \lambda_{2}\right)$ for $m_{A}, m_{\chi} \ll M_{*}$. 
The masses of isospin doublet and color triplet Higgses are obtained from the superpotential

$$
W_{H}=M_{i j} H_{i} H_{j}+\lambda_{H} A H_{1} H_{2}+\lambda_{\chi}^{i} H_{i} \chi \chi+\bar{\lambda}_{\chi}^{i} H_{i} \bar{\chi} \bar{\chi},
$$

and the mass term is

$$
\begin{aligned}
& \left(\begin{array}{lll}
H_{1}^{\mathbf{5}} & H_{2}^{\mathbf{5}} & \bar{\chi}^{\mathbf{5}}
\end{array}\right) M_{H_{D, T}}\left(\begin{array}{c}
H_{1}^{\overline{5}} \\
H_{2}^{\overline{5}} \\
\chi^{\overline{\mathbf{5}}}
\end{array}\right), \\
& M_{H_{D, T}}=\left(\begin{array}{ccc}
M_{11} & M_{12}+\lambda_{H} A_{D, T} & \lambda_{\chi}^{1} v_{R} \\
M_{12}-\lambda_{H} A_{D, T} & M_{22} & \lambda_{\chi}^{2} v_{R} \\
\bar{\lambda}_{\chi}^{1} \bar{v}_{R} & \bar{\lambda}_{\chi}^{2} \bar{v}_{R} & m_{\chi}+\frac{\kappa}{\sqrt{5}}\left(-3 a_{1}+\sqrt{\frac{2}{3}} c_{D, T} a_{24}\right)
\end{array}\right),
\end{aligned}
$$

where

$$
A_{D, T}=\frac{1}{\sqrt{5}}\left(a_{1}+\frac{1}{\sqrt{6}} c_{D, T} a_{24}\right)
$$

and $\left(c_{D}, c_{T}\right)=(3,-2)$. The doublet-triplet splitting needs fine-tuning. Without loss of generality, $\lambda_{\chi}^{1}$ is set to zero by a rotation of $\left(H_{1}, H_{2}\right)$. In this basis, by the fine-tuning $M_{11}=M_{12}+\lambda_{H} A_{D}=0$, we have one pair of doublets massless. $H_{1}$ in this basis should dominantly give the large top quark Yukawa coupling. The mass of the corresponding triplet is roughly $\sim 5 / 3 \lambda_{H} A_{D}$ for $\left|a_{1}\right|,\left|v_{R}\right| \ll\left|a_{24}\right|$.

\section{B Renormalizable model obtained by employing $54_{\mathrm{H}}$}

In the main text, we have considered the model with $\mathbf{4 5}_{\mathrm{H}}+\mathbf{1 6} \mathbf{6}_{\mathrm{H}}+\overline{\mathbf{1 6}}_{\mathrm{H}}$ and with nonrenormalizable quartic terms of $\mathbf{4} \mathbf{5}_{\mathrm{H}}$. In this appendix, for readers who prefer renormalizable models, we show that a renormalizable superpotential with $\mathbf{5} \mathbf{4}_{\mathrm{H}}$ (whose SM singlet component is denoted by $E$ ) can also provide the wanted vacua where $\left|a_{24}\right| \gg\left|a_{1}\right|,\left|v_{R}\right|$ (and $\left|a_{24}\right| \gg|E|$ ).

The superpotential for the SM singlets is

$$
\begin{aligned}
\left.W\right|_{\text {SM singlets }}= & m_{\chi} v_{R} \bar{v}_{R}+\frac{1}{2} m_{A}\left(a_{1}^{2}+a_{24}^{2}\right)+\frac{1}{2} m_{E} E^{2}+\sqrt{5} \kappa a_{1} v_{R} \bar{v}_{R} \\
& +\frac{\kappa_{1}}{3} E^{3}+\kappa_{2} E\left(\sqrt{6} a_{1} a_{24}+\frac{1}{2} a_{24}^{2}\right)
\end{aligned}
$$

From the $F$-flat conditions, we obtain

$$
a_{1}=-\frac{m_{\chi}}{\sqrt{5} \kappa}, \quad a_{24}=\sqrt{\frac{6}{5}} \frac{m_{\chi}}{\kappa} a, \quad v_{R} \bar{v}_{R}=\frac{m_{\chi} m_{A}}{5 \kappa^{2}} \frac{6 a^{2}+a-1}{a-1}, \quad E=-\frac{m_{A}}{\kappa_{2}} \frac{a}{a-1},
$$

where $a$ is a solution of the following equation:

$$
m_{E}=\kappa_{1} \frac{m_{A}}{\sqrt{5} \kappa} \frac{a}{a-1}+\frac{3 \kappa_{2}^{2} m_{\chi}^{2}}{5 \kappa^{2} m_{A}}(a-1)(a-2) .
$$


Vacua with $|E|,\left|a_{1}\right|,\left|v_{R}\right| \ll\left|a_{24}\right|$ are obtained by assuming $m_{\chi}, m_{A} \ll m_{E}$, which gives

$$
a_{24} \simeq \frac{\sqrt{2 m_{A} m_{E}}}{\kappa_{2}}
$$

The $\mathbf{5} \mathbf{4}_{\mathrm{H}}$ is decomposed as $\mathbf{5} \mathbf{4}_{\mathrm{H}}=\mathbf{2 4}+\mathbf{1 5}+\overline{\mathbf{1 5}}$ under $\mathrm{SU}(5)$. The mass matrices of the adjoint representations after $\mathrm{SU}(5)$ breaking are

$$
\left(\begin{array}{cc}
m_{A}-2 \kappa_{2} E & -2 \kappa_{2} a_{24}+\sqrt{6} \kappa_{2} a_{1} \\
-2 \kappa_{2} a_{24}+\sqrt{6} \kappa_{2} a_{1} & m_{E}-4 \kappa_{1} E
\end{array}\right)
$$

for $(\mathbf{8}, \mathbf{1}, 0)$ and

$$
\left(\begin{array}{cc}
m_{A}+3 \kappa_{2} E & 3 \kappa_{2} a_{24}+\sqrt{6} \kappa_{2} a_{1} \\
3 \kappa_{2} a_{24}+\sqrt{6} \kappa_{2} a_{1} & m_{E}+6 \kappa_{1} E
\end{array}\right)
$$

for $(\mathbf{1}, \mathbf{3}, 0)$. For $a \gg 1, \kappa_{2} E \simeq-m_{A}$, and we obtain the masses of the lighter adjoint fields (using $\left.2 m_{A} m_{E} \simeq\left(\kappa_{2} a_{24}\right)^{2}\right)$ as $M_{8} \simeq(3-4 \times 2) m_{A}=-5 m_{A}$ and $M_{3} \simeq(-2-9 \times 2) m_{A}=$ $-20 m_{A}$, and hence $M_{8}: M_{3} \simeq 1: 4$. We have thus verified that the mass ratio $M_{8}: M_{3}$ is the same as the model with the non-renormalizable terms, which is obtained by integrating out $54_{\mathrm{H}}$.

Open Access. This article is distributed under the terms of the Creative Commons Attribution License (CC-BY 4.0), which permits any use, distribution and reproduction in any medium, provided the original author(s) and source are credited.

\section{References}

[1] P. Langacker, Grand unified theories and proton decay, Phys. Rept. 72 (1981) 185 [INSPIRE].

[2] Super-Kamiokande collaboration, Search for proton decay via $p \rightarrow e^{+} \pi^{0}$ and $p \rightarrow \mu^{+} \pi^{0}$ in 0.31 megaton-years exposure of the Super-Kamiokande water Cherenkov detector, Phys. Rev. D 95 (2017) 012004 [arXiv:1610.03597] [InSPIRE].

[3] Hyper-Kamiokande collaboration, Hyper-Kamiokande design report, arXiv:1805.04163 [INSPIRE].

[4] S. Dimopoulos and H. Georgi, Softly broken supersymmetry and SU(5), Nucl. Phys. B 193 (1981) 150 [inSPIRE].

[5] S. Dimopoulos, S. Raby and F. Wilczek, Supersymmetry and the scale of unification, Phys. Rev. D 24 (1981) 1681 [INSPIRE].

[6] N. Sakai, Naturalness in supersymmetric GUTs, Z. Phys. C 11 (1981) 153 [INSPIRE].

[7] S. Weinberg, Supersymmetry at ordinary energies. 1. Masses and conservation laws, Phys. Rev. D 26 (1982) 287 [InSPIRE].

[8] N. Sakai and T. Yanagida, Proton decay in a class of supersymmetric grand unified models, Nucl. Phys. B 197 (1982) 533 [InSPIRE].

[9] Super-Kamiokande collaboration, Search for proton decay via $p \rightarrow \nu K^{+}$using 260 kiloton.year data of Super-Kamiokande, Phys. Rev. D 90 (2014) 072005 [arXiv: 1408.1195] [INSPIRE]. 
[10] K. Tobe and J.D. Wells, Gravity assisted exact unification in minimal supersymmetric SU(5) and its gaugino mass spectrum, Phys. Lett. B 588 (2004) 99 [hep-ph/0312159] [INSPIRE].

[11] B. Bajc, P. Fileviez Perez and G. Senjanović, Minimal supersymmetric SU(5) theory and proton decay: where do we stand?, in Beyond the desert: accelerator, non-accelerator and space approaches into the next millennium. Proceedings, $3^{\text {rd }}$ International Conference on particle physics beyond the Standard Model, Oulu, Finland, 2-7 June 2002, pg. 131 [hep-ph/0210374] [INSPIRE].

[12] B. Dutta, Y. Mimura and R.N. Mohapatra, Proton decay and flavor violating thresholds in SO(10) models, Phys. Rev. Lett. 100 (2008) 181801 [arXiv:0712.1206] [INSPIRE].

[13] J. Hisano, T. Kuwahara and N. Nagata, Grand unification in high-scale supersymmetry, Phys. Lett. B 723 (2013) 324 [arXiv:1304.0343] [INSPIRE].

[14] B. Bajc, J. Hisano, T. Kuwahara and Y. Omura, Threshold corrections to dimension-six proton decay operators in non-minimal SUSY SU(5) GUTs, Nucl. Phys. B 910 (2016) 1 [arXiv: 1603.03568] [INSPIRE].

[15] J. Ellis, J.L. Evans, A. Mustafayev, N. Nagata and K.A. Olive, The super-GUT CMSSM revisited, Eur. Phys. J. C 76 (2016) 592 [arXiv: 1608.05370] [inSPIRE].

[16] S. Pokorski, K. Rolbiecki and K. Sakurai, Proton decay testing low energy supersymmetry with precision gauge unification, Phys. Rev. D 97 (2018) 035027 [arXiv:1707.06720] [INSPIRE].

[17] N. Haba, Y. Mimura and T. Yamada, Proton lifetime upper bound in non-SUSY SU(5) GUT, Phys. Rev. D 99 (2019) 075018 [arXiv: 1812.08521] [INSPIRE].

[18] P. Nath and R.M. Syed, Complete cubic and quartic couplings of 16 and bar-16 in $\mathrm{SO}(10)$ unification, Nucl. Phys. B 618 (2001) 138 [hep-th/0109116] [INSPIRE].

[19] D.R.T. Jones, The two loop $\beta$-function for a $G_{1} \times G_{2}$ gauge theory, Phys. Rev. D 25 (1982) 581 [INSPIRE].

[20] M.E. Machacek and M.T. Vaughn, Two loop renormalization group equations in a general quantum field theory. 1. Wave function renormalization, Nucl. Phys. B 222 (1983) 83 [INSPIRE].

[21] Particle Data Group collaboration, Review of particle physics, Phys. Rev. D 98 (2018) 030001 [inSPIRE].

[22] P. Nath and P. Fileviez Perez, Proton stability in grand unified theories, in strings and in branes, Phys. Rept. 441 (2007) 191 [hep-ph/0601023] [INSPIRE].

[23] Y. Aoki, T. Izubuchi, E. Shintani and A. Soni, Improved lattice computation of proton decay matrix elements, Phys. Rev. D 96 (2017) 014506 [arXiv: 1705.01338] [INSPIRE].

[24] J. Hisano, D. Kobayashi, Y. Muramatsu and N. Nagata, Two-loop renormalization factors of dimension-six proton decay operators in the supersymmetric Standard Models, Phys. Lett. B 724 (2013) 283 [arXiv:1302.2194] [INSPIRE].

[25] J. Hisano, H. Murayama and T. Yanagida, Nucleon decay in the minimal supersymmetric SU(5) grand unification, Nucl. Phys. B 402 (1993) 46 [hep-ph/9207279] [InSPIRE]. 